\title{
EFECTIVIDAD DEL 3,4- DIMETILPIRAZOLFOSFATO (DMPP) EN NARANJO DULCE EN EL NORESTE ARGENTINO ${ }^{1}$
}

\author{
VÍCTOR ANTONIO RODRÍGUEZ ${ }^{2}$, PAULA ALAYÓN LUACES ${ }^{3}$, ANALÍA BEATRIZ PÍCCOLI ${ }^{4}$, \\ SILVIA MATILDE MAZZA ${ }^{5}$, GLORIA CRISTINA MARTÍNEZ ${ }^{6}$
}

RESUMEN - El objetivo del trabajo fue evaluar el efecto del 3,4-dimetilpirazol fosfato (DMPP) en la eficiencia de la fertilización amoniacal, en naranjo dulce [Citrus sinensis (L.) Osbeck] 'Valencia late' sobre suelo arenoso, en la provincia de Corrientes, Argentina. Durante tres campañas se trabajó en un huerto de naranjo 'Valencia late' injertado sobre lima de Rangpur (Citrus limonia, Osbeck), sobre un suelo tipo Udipsamment alfico. Se utilizó un Diseño de Bloques Completos al Azar con cuatro repeticiones, parcela útil una planta

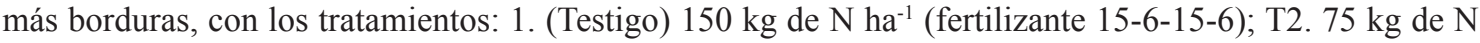
$\mathrm{ha}^{-1}$ (sulfonitrato de amonio (SNA) $26 \% \mathrm{~N}$ ) tratado con DMPP (0,8\% respecto al $\mathrm{N}$ amoniacal); T3. 150 $\mathrm{kg}$ de $\mathrm{N} \mathrm{ha}^{-1}$ (SNA $\left.26 \% \mathrm{~N}\right)$, tratado con DMPP $(0,8 \%$ respecto al $\mathrm{N}$ amoniacal). En el otoño se tomaron muestras foliares de ramas fructíferas, determinándose contenido de N, P y K. En la cosecha se determinó producción por planta $(\mathrm{kg})$ y se evaluó calidad de frutos. Los resultados obtenidos permiten establecer que en suelos arenosos, el agregado de DMPP mejora la eficiencia de la fertilización nitrogenada. Con igual aporte nitrogenado, se incrementan los rendimientos y las concentraciones foliares de $\mathrm{N}$ y con la mitad de la dosis se logran rendimientos equivalentes al testigo.

Palabras clave: Inhibidores nitrificación - Citrus sinensis - fertilización - productividad.

\section{3,4-DIMETHYLPYRAZOLE PHOSPHATE (DMPP) EFFICIENCY IN SWEET ORANGE IN ARGENTINA NORTHEAST}

\begin{abstract}
The aim of this study was to evaluate the 3,4-dimethylpirazol phosphate (DMPP) effect on the efficiency of ammonium-N fertilization on sandy soil of Corrientes, Argentina. The experiment was carried out with sweet orange [Citrus sinensis (L.) Osbeck] 'Valencia late' grafted on Rangpur lime (Citrus limonia, Osbeck) rootstock under field conditions during three consecutive years, the orchard was established on an alfic Udipsamment soil. A randomized complete block statistical design with four replications was used and the treatments were: 1 . (Control) $150 \mathrm{~kg} \mathrm{~N} \mathrm{ha}^{-1}$ (fertilizer 15-6-15-6); $2.75 \mathrm{~kg} \mathrm{~N} \mathrm{ha}^{-1}$ (ammonium sulphonitrate (SNA) $26 \% \mathrm{~N}$ ) treated with DMPP $\left(0.8 \%\right.$ compared to ammonia $\mathrm{N}$ ); $3.150 \mathrm{~kg} \mathrm{~N}^{-1}$ (SNA $26 \% \mathrm{~N})$, treated with DMPP $(0.8 \%$ compared to ammonia $\mathrm{N})$. Leaf samples from fruitful branches were taken in autumn and the amount of N, P and $\mathrm{K}$ were analysed. At harvest, yield per plant was measured $(\mathrm{kg})$ and quality of fruits was assessed. The results obtained demonstrate that in sandy soils, the addition of DMPP improves the efficiency of nitrogen fertilization. Identical nitrogen supply, increase yields and foliar concentrations of $\mathrm{N}$ and with the half $\mathrm{N}$ dosage, control equivalent yields were achieved.
\end{abstract}

Index terms: nitrification inhibitor - Citrus sinensis - fertilization - productivity.

\footnotetext{
'(Trabalho 073-11). Recebido em: 08-02-2011. Aceito para publicação em: 09-09-2011.

${ }^{2}$ Ing.Agr. (Esp.) V.A. Rodríguez. Sargento Cabral 2131 - W3402BKG - Corrientes, Argentina. cocorodriguez@ciudad.com ${ }^{3}$ Ing. Agr. P. Alayón Luaces. Sargento Cabral 2131 - W3402BKG - Corrientes, Argentina. p_alayon@agr.unne.edu.ar ${ }^{4}$ Ing. Agr. A.B. Píccoli. Sargento Cabral 2131 -W3402BKG - Corrientes, Argentina. abpicoli@agr.unne.edu.ar ${ }^{5}$ Ing. Agr. (Dra.) S. M. Mazza. Sargento Cabral 2131 - W3402BKG- Corrientes, Argentina. smmazza@agr.unne.edu.ar ${ }^{6}$ Ing. Agr. (Mgter.) G. C. Martínez. Sargento Cabral 2131 - W3402BKG - Corrientes, Argentina. gloriam@agr.unne.edu.ar
} 
La demanda de frutos de calidad es una realidad creciente en los mercados mundiales y el eficiente manejo de la fertilización, principalmente nitrogenada, es clave para lograr este objetivo, tanto por razones económicas como ecológicas. En las plantaciones citrícolas del mundo, los planes de fertilización utilizan mayoritariamente fuentes de forma amoniacal para el aporte de nitrógeno (PALACIOS, 2005). Los suelos del nordeste argentino son arenosos, con bajo porcentaje de materia orgánica y escasa capacidad de retención de agua y sales minerales, implicando altas pérdidas de nitrógeno por lixiviación y desnitrificación. Las altas concentraciones de $\mathrm{NO}_{3}^{-}$, elevada humedad, bajo contenido de $\mathrm{O}_{2} \mathrm{y}$ $\mathrm{pH}$ elevado facilitan la acción de microorganismos (Pseudomonas sp., Bacillus sp., Paracocus sp.) que transforman el $\mathrm{NO}_{3}^{-}$en monóxido de dinitrógeno $\left(\mathrm{N}_{2} \mathrm{O}\right)$ o nitrógeno molecular $\left(\mathrm{N}_{2}\right)$, que finalmente se difunden hacia la atmósfera. Estos compuestos gaseosos, además de suponer una pérdida de nitrógeno, son nocivos para la capa de ozono y favorecen el calentamiento de la atmósfera (LINZMEIER et al., 2001). Los inhibidores de la nitrificación son compuestos que, añadidos a los fertilizantes, inhiben las bacterias Nitrosomonas y retrasan la oxidación bacteriana de $\mathrm{NH}_{4}^{+}$a nitrito $\left(\mathrm{NO}_{2}^{-}\right)$en el suelo (primer paso de la nitrificación), durante un cierto tiempo, e imposibilitan su transformación final en $\mathrm{NO}_{3}$. Se ha demostrado que cuando existe un suministro combinado de ambas formas de nitrógeno $\left(\mathrm{NO}_{3}^{-} \mathrm{y} \mathrm{NH}_{4}^{+}\right)$, se obtienen mayores tasas de crecimiento y rendimiento (GOOS et al., 1999) debido a que la absorción de $\mathrm{NH}_{4}^{+}$significa un ahorro energético al interior de la planta y además ocasiona un descenso del $\mathrm{pH}$ de la zona radicular, que puede contribuir a la absorción de otros nutrientes. En condiciones de campo la aplicación de $\mathrm{NH}_{4}^{+}$junto con un inhibidor de la nitrificación permite suministrar $\mathrm{NH}_{4}^{+} \mathrm{y} \mathrm{NO}_{3}^{-}$conjuntamente (aún con inhibidor de la nitrificación existen pequeñas concentraciones de $\mathrm{NO}_{3}^{-}$), lo que favorece mayores tasas de crecimiento y rendimiento respecto al suministro único de abonos nítricos (LITE et al., 2005). Investigaciones sobre compuestos capaces de retrasar la oxidación biológica de $\mathrm{NH}_{4}^{+} \mathrm{a} \mathrm{NO}_{3}^{-}$han demostrado que la acción de inhibidores de la nitrificación como el 3,4-dimethylpirazol fosfato (DMPP), son altamente efectivos con numerosas ventajas para el ambiente por poseer muy bajo riesgo de lixiviación (FETTWEIS et al., 2001), y disminuir la cantidad de $\mathrm{N}_{2} \mathrm{O}$ liberado (WEISKE et al., 2001). Bañuls et al. (2000), demostraron que el DMPP incorporado junto con el sulfato de amonio en riego por goteo, mejoró la absorción del nitrógeno por parte de los cítricos. El objetivo de este trabajo fue evaluar el efecto del
DMPP sobre la eficiencia de la fertilización amoniacal en naranjo dulce [Citrus sinensis (L.) Osbeck] 'Valencia late', implantada en un suelo arenoso, en las condiciones edafoclimáticas de la provincia de Corrientes, Argentina. El trabajo de campo se realizó durante las campañas 2002/03, 2003/04 y 2004/05, en un huerto comercial de naranjo dulce 'Valencia late' injertado sobre lima de Rangpur (Citrus limonia, Osbeck), implantada en 1986, con una densidad de 250 plantas $\mathrm{ha}^{-1}$, en un suelo Udipsamment alfico del Departamento de Concepción (28 $8^{\circ} 16^{\prime} \mathrm{S} ; 58^{\circ} 05^{\prime}$ $\mathrm{W} ; 51 \mathrm{msnm}$ ), provincia de Corrientes-Argentina. Se utilizó un Diseño en Bloques Completos al Azar con cuatro repeticiones, parcela útil una planta más borduras, para probar los siguientes tratamientos:

1. (T1) (testigo) $150 \mathrm{~kg} \mathrm{de} \mathrm{N} \mathrm{ha}^{-1}$, utilizando el $40 \mathrm{~kg} \mathrm{ha}^{-1}$ del fertilizante $15 \mathrm{~N}-6 \mathrm{P}_{2} \mathrm{O}_{5}-15 \mathrm{~K}_{2} 0-6$ $\mathrm{MgO}$; corresponde a la dosis de $\mathrm{N}$ de uso corriente en la región citrícola argentina y equivalente a la dosis máxima probada.

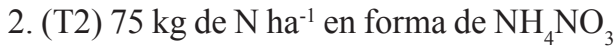
$\left(\mathrm{NH}_{4}\right)_{2} \mathrm{SO}_{4}$ (sulfonitrato de amonio; SNA) $(26 \% \mathrm{~N}$; $19 \%$ en forma amoniacal y $7 \%$ en forma de nitratos), tratado con DMPP $(0,8 \%$ respecto al $\mathrm{N}$ amoniacal).

3. (T3) $150 \mathrm{~kg}^{\mathrm{de}} \mathrm{N} \mathrm{ha}^{-1}$ en forma de SNA $(26 \% \mathrm{~N} ; 19 \%$ en forma amoniacal y $7 \%$ en forma de nitratos), tratado con DMPP $(0,8 \%$ respecto al $\mathrm{N}$ amoniacal).

Todos los tratamientos recibieron idéntico aporte fósforo $(\mathrm{P})$ y potasio $(\mathrm{K})$, y las dosis se aplicaron la mitad en diciembre y el resto en marzo. En el otoño se muestrearon 100 hojas de ramas fructíferas, de siete meses correspondiente a la brotación de primavera provenientes de los cuatro puntos cardinales de la planta, en la que se determinaron el contenido de nitrógeno $(\mathrm{N})$, por el método de Kjeldhal; P por espectrometría de absorción molecular (método Murphy-Riley) y K por espectrometría de absorción atómica (KALRA, 1998). En septiembre, al momento de la cosecha se determinó producción total de frutos por planta $(\mathrm{kg})$ y para evaluar calidad, se realizó un muestreo aleatorio de diez frutos por unidad experimental, determinándose diámetro ecuatorial $(\mathrm{mm})$, coloración de cáscara mediante escala visual de 1 a 9 (1 verde intenso y 9 naranja intenso), porcentaje de jugo, acidez (en mg de ácido cítrico) y ratios (Palacios, 2005). Se realizó Análisis de la Variancia y posterior test de Tukey para las variables cuantitativas y Análisis de la Variancia no paramétrico de Kruskal Walis para color (STEEL; TORRIE, 1992). Para todas las variables cuantitativas, las interacciones entre tratamientos $y$ años resultaron no significativas $(\mathrm{p}>0,05)$, por lo que 
se realizó el análisis conjunto de todas las campañas. La Tabla 1 presenta los rendimientos promedio de los tratamientos probados, en el conjunto de las tres campañas (coeficientes de variación correspondientes a las variables rendimiento: $14,47 \%$; diámetro: 2,77\%; jugo: 5,35\%; Brix: 7,77\%; acidez: $8,39 \%$ y ratio: $10,77 \%)$. Se detecta mayor producción total de frutos en el tratamiento con la mayor dosis de SNA+DMPP (T3), en relación con la menor dosis (T2) y el testigo; y con T2 (50\% de la dosis testigo) se logró una producción similar al testigo. Esto concuerda con lo informado por Handell y Zerulla (2001), quienes encontraron que la cosecha en árboles frutales tratados con fertilizantes nitrogenados + DMPP fueron iguales o superiores que aquellos recibieron aplicaciones estándares con fertilizantes sin suplemento del inhibidor. Si bien el promedio de diámetro de frutos de los tres tratamientos se encuentra dentro de valores aceptables para el mercado internacional, frutos no menores a $60 \mathrm{~mm}$ de diámetro, con $\mathrm{T} 3$ se lograron menores diámetros en relación con el testigo por la mayor cantidad de $\mathrm{kg}$ de frutos cosechados; en tanto que $\mathrm{T} 2$ no se diferenció de los otros dos. No se detectó efecto de los tratamientos en las variables de calidad interna y externa de los frutos, resultados coincidentes con lo citado por Quiñones et al. (2009) para \% jugo, acidez y Ratios. En la Figura 1 se aprecian los resultados de cosecha de las tres campañas en estudio. En ella se puede observar que en las campañas 2002/03 y 2004/05, los rendimientos de los tratamientos con DMPP superaron al testigo, sin embargo en esta última campaña es probable que los mismos se vieran afectados por las escasas precipitaciones registradas en la primavera 2004. En la campaña 2003/04, los rendimientos de todos los tratamientos se elevaron considerablemente, posiblemente debido a las abundantes lluvias de la primavera del 2003, que favorecieron un buen cuajado de frutos en todas las plantas del ensayo, poniéndose de relevancia los excelentes rendimientos del tratamiento con mayor dosis de DMPP. En cuanto al diámetro de los frutos (Figura 2), en las campañas 2002/03 y 2004/05, los rangos de tamaños se encuentran entre las mejores categorías para mercados de frutos frescos. En la campaña 2003/04, en correspondencia con los más altos rendimientos, los valores se encuentran en los límites inferiores de las categorías aceptadas para mercado de frutos frescos. Estos resultados ponen en evidencia el rol del DMPP, en razón de que con idéntica dosis de $\mathrm{N}$ por hectárea (testigo y T3), se obtienen mejores rendimientos con el agregado de DMPP. Esto concuerda con lo reportado por Serna et al. (2000) y Quiñones et al. (2009), que el agregado de DMPP al SNA mejora la eficiencia de la fertilización nitrogenada. Puede explicarse, además, por lo encontrado por Pasda et al. (2001), quienes señalan que el agregado de DMPP produce efecto positivo sobre los rendimientos de cultivos frutihortícolas en suelos arenosos y Wu et al. (2007), que obtuvieron mejor respuesta a la aplicación de N + DMPP en suelos arenosos que en suelos arcillosos. En la Tabla 2 se presentan los contenidos foliares de N, P y K por tratamiento, promedio de las tres campañas (coeficientes de variación correspondientes a las variables nitrógeno: $7,10 \%$; fósforo: $11,18 \%$ y potasio: $12,94 \%$ ). Con el T3 se detectan mayores contenidos de $\mathrm{N}$ relación con el testigo, al igual que lo encontrado por Quiñones et al. (2009) poniendo en evidencia la eficiencia del DMPP en el aprovechamiento del $\mathrm{N}$, en tanto que $\mathrm{T} 2$ presentó un comportamiento intermedio y no se diferenció de los otros dos tratamientos. No se detectó efecto de los tratamientos en los contenidos foliares de P y K. Las concentraciones foliares de N y $P$ se encuentran dentro de los rangos considerados normales para la especie y variedad en estudio no así los valores de $\mathrm{K}$ que se hallan dentro del rango de deficiencia (PALACIOS, 2005).

En función de los resultados obtenidos en este trabajo se puede concluir que en suelos arenosos el tratamiento con $\mathrm{N}\left(150 \mathrm{~kg} \mathrm{ha}^{-1}\right)$ suplementado con DMPP mejora la eficiencia de la fertilización nitrogenada para las variables rendimiento y concentración foliar de $\mathrm{N}$. 
TABLA 1 - Rendimientos obtenidos a la cosecha, promedio en el conjunto de las tres campañas, en $\mathrm{kg}$ planta $^{-1}$ (Rend), diámetro ecuatorial de los frutos en mm (Diam), porcentaje de jugo (Jugo), grados Brix (Brix), acidez (Acidez), ratios (Ratio) y color de cáscara (Color), por tratamientos y resultados del test de Tukey.

\begin{tabular}{cccccccccc}
\hline Tratamiento & Rend & Diam & Jugo & Brix & Acidez & Ratio & Color \\
\hline T1 - 150 kg. de N ha-1 & 144,33 a & 66,08 a & 51,95 a & 10,16 a & 0,97 & a & 9,77 & a & 5,20 \\
T2 - 75 kg de N ha
\end{tabular}

Letras iguales en las columnas indican diferencias no significativas de acuerdo con el test de Tukey $\alpha=0,05$

TABLA 2 - Concentraciones foliares de nitrógeno $(\mathrm{N})$, fósforo $(\mathrm{P})$ y potasio $(\mathrm{K})$, expresadas en $\mathrm{g} \mathrm{kg}^{-1}$, por tratamiento y resultados del test de Tukey $(\mathrm{p}>0,05)$, promedio de los tres años en estudio

\begin{tabular}{|c|c|c|c|c|c|c|c|}
\hline Tratamiento & $\mathrm{N}$ & & & $\mathrm{P}$ & & $\mathrm{K}$ & \\
\hline $\mathrm{T} 1$ - $150 \mathrm{~kg}$ de $\mathrm{N} \mathrm{ha}^{-1}$ & 0,193 & $\mathrm{a}$ & & 0,013 & $\mathrm{a}$ & 0,052 & $\mathrm{a}$ \\
\hline T2 - $75 \mathrm{~kg}$ de $\mathrm{N} \mathrm{ha}^{-1}+\mathrm{DMPP}$ & 0,206 & $\mathrm{a}$ & $b$ & 0,014 & $\mathrm{a}$ & 0,055 & $\mathrm{a}$ \\
\hline T3 - $150 \mathrm{~kg}$ de $\mathrm{N} \mathrm{ha}^{-1}+$ DMPP & 0,218 & & $\mathrm{~b}$ & 0,015 & $\mathrm{a}$ & 0,057 & $\mathrm{a}$ \\
\hline
\end{tabular}

Letras iguales en las columnas indican diferencias no significativas de acuerdo con el test de Tukey $\alpha=0,05$

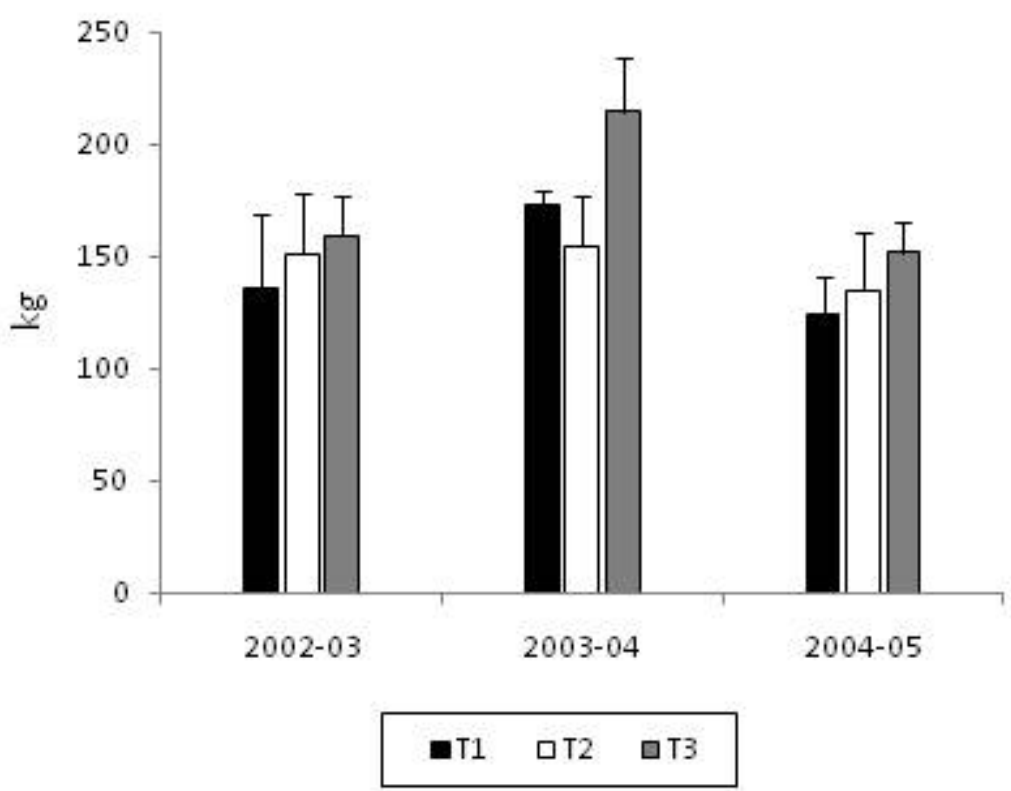

FIGURA 1 - Producción total de frutos por planta $(\mathrm{kg})$, promedio y error estándar de tres campañas por tratamiento. 


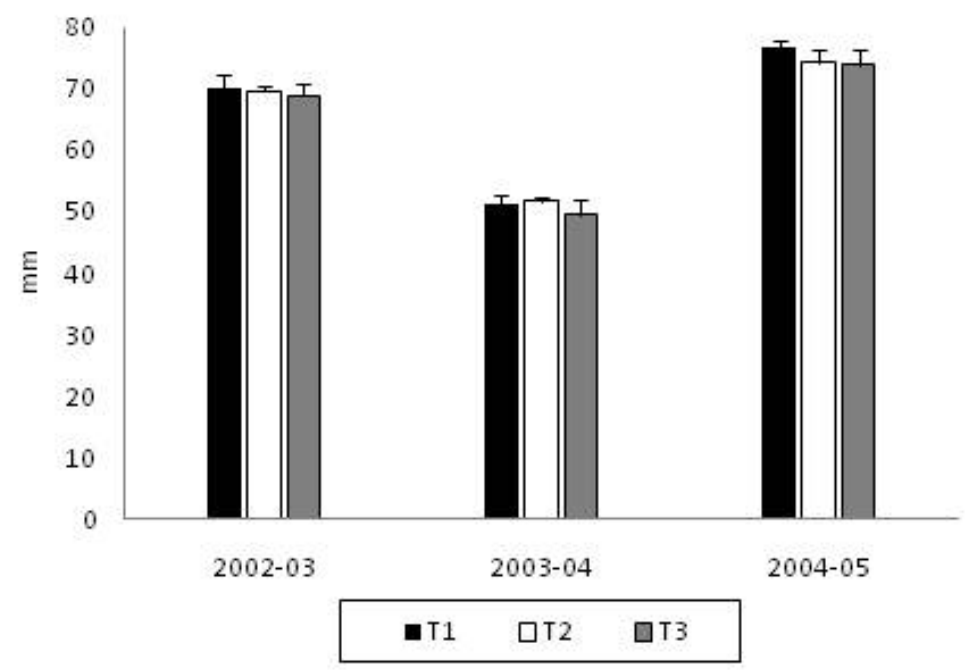

FIGURA 2 - Diámetro de frutos ( $\mathrm{mm})$, promedio y error estándar de tres campañas por tratamiento.

\section{REFERENCIAS}

BAÑULS, J.; SERNA, M.D.; QUIÑONES, A.; MARTIN, B.; PRIMO MILLO, E.; LEGAZ, F. Optimización de la fertilización nitrogenada con el inhibidor de la nitrificación (DMPP) con riego por goteo en cítricos. Levante Agrícola, Valência, v.351, p.117-121, 2000.

FETTWEIS, U.; MITTELSTAEDT, W. Lysimeter experiments on the translocation of the carbon-14labelled nitrification inhibitor 3,4-dimethylpyrazole phosphate (DMPP) in a gleyic cambisol. Biology and Fertility of Soils, Berlin, v.34, p.126-130, 2001.

GOOS, R.J.; SCHIMELFENIG, J.A.; BOCK, B.R.; JOHNSON, B.E. Response of spring wheat to nitrogen fertilizers of different nitrification rates. Agronomy Journal, Madison, v.91, p.287-293, 1999.

HÄHNDEL, R.; ZERULLA, W. Effects of ammonium-stabilized n-fertilizers on yield and quality of vegetables. Acta Horticulturae, The Hague, v.563, p.81-86, 2001. Disponível em: $<$ http://www.actahort.org/books/563/563 9.htm>.

KALRA, P.Y. Handbook of reference methods for plant analysis. Boca Raton: CRC Press, 1998. 300 p.
LINZMEIER, W.; GUTSER, R.; SCHMIDHALTER, U. Nitrous oxide emission from soil and from a nitrogen-15-labelled fertilizer with the new nitrification inhibitor 3,4-dimethylpyrazole phosphate (DMPP). Biology and Fertility of Soils, Berlin, v.34, p.103-108, 2001.

LITE, A. L.; MUÑOZ-GUERRA REVILLA, L. M. Fertilización eco-eficiente del olivo y disminución de la contaminación por nitratos mediante inhibidores de la nitrificación. In: FORO DEL OLIVAR Y DEL MEDIO AMBIENTE, 2005. (Comunicación Técnica OLI-21).

PALACIOS, J. Citricultura. Buenos Aires: Editorial Hemisferio Sur, 2005. 518 p.

PASDA, G.; HÄHNDEL, R.; ZERULLA, W. The new nitrification inhibitor DMPP (ENTEC () - Effects on yield and quality of agricultural and horticultural crops. In: HORST, W.J. (Ed.). Plant nutrition: food security and sustainability of agro-ecosystems. through basic and applied research. Dordrecht: Kluwer Academic Publications, 2001. p.758-759. 2001.

QUIÑONES, A.; MARTÍNEZ-ALCÁNTARA, B.; CHI-BACAB, U.; LEGAZ, F. Improvement of N fertilization by using the nitrification inhibitor DMPP in drip-irrigated citrus trees. Spanish Journal of Agricultural Research, Lahore, v.7, n.1, p.190199, 2009. 
SERNA, M.D.; BAÑULS, J.; QUIÑONES, A.; PRIMO-MILLO, E.; LEGAZ, Y F. Evaluation of 3,4-dimethylpyrazole phosphate as a nitrification inhibitor in a Citrus cultivated soil. Biology and Fertility of Soil, Berlin, v.32, p. 41-46, 2000.

STEEL, R.G.D.; J.H. TORRIE. Bioestadística: principios y procedimientos. 2. ed. México: Mc Graw Hill, 1992. 622 p.

WEISKE, A.; BENCKISER, G.; OTTOW, J.C.G. The new nitrification inhibitor DMPP - effects on gaseous emissions $(\mathrm{N} 2 \mathrm{O}, \mathrm{CO} 2 \mathrm{CH} 4)$ from soil under field conditions. In: HORST, W.J. (Ed.). Plant nutrition: food security and sustainability of agro-ecosystems through basic and applied research. Dordrecht: Kluwer Academic Publications, 2001. p.766-767.
WISSEMEIER, A.H. 3,4- Dimethylpyrazole phosphate (DMPP) - a new nitrification inhibitor for agriculture and horticulture. Biology and Fertility of Soil, Berlin, v.34, p.79, 2001.

WU, S.F.; WU, L.H.; SHI, Q.W.; WANG, Z.Q.; CHEN, X.Y.; LI, Y.S.Effects of a new nitrification inhibitor 3,4-dimethylpyrazole phosphate (DMPP) on nitrate and potassium leaching in two soils. Journal of Environmental Science, New York, v.19, n.7, p.841-847, 2007. 